\title{
Diagnostic Value of the Thoracic Ultrasonography Compared to Conventional Chest X-Rays in Pneumonia for Children between 0 to 15 Years: Case Study in Two Hospitals in Yaoundé
}

\author{
Seme Engoumou Ambroise Merci1,2, Mbede Maggy1,3, Awana Armel Philippe ${ }^{1,4}$, \\ Bilounga Ndengue Priscille Edith', Onguene Julienne ${ }^{1,5}$, Zeh Odile Fernande ${ }^{1,5}$ \\ ${ }^{1}$ Faculty of Medicine and Biomedical Sciences, The University of Yaoundé I, Yaounde, Cameroon \\ ${ }^{2}$ Center for Research and Applied Endoscopic Surgery in Reproduction Health, Yaounde, Cameroon \\ ${ }^{3}$ Yaoundé Central Hospital, Yaounde, Cameroon \\ ${ }^{4}$ Yaoundé Jamot Hospital, Yaounde, Cameroon \\ ${ }^{5}$ Yaoundé Gyneco-Obstetrical and Pediatric Hospital, Yaounde, Cameroon \\ Email: ambroise.seme@gmail.com
}

How to cite this paper: Merci, S.E.A., Maggy, M., Philippe, A.A., Edith, B.N.P., Julienne, O. and Fernande, Z.O. (2019) Diagnostic Value of the Thoracic Ultrasonography Compared to Conventional Chest $\mathrm{X}$-Rays in Pneumonia for Children between 0 to 15 Years: Case Study in Two Hospitals in Yaoundé. Open Journal of Radiology, 9 , $10-19$.

https://doi.org/10.4236/ojrad.2019.91002

Received: December 2, 2018

Accepted: January 11, 2019

Published: January 14, 2019

Copyright $\odot 2019$ by author(s) and Scientific Research Publishing Inc. This work is licensed under the Creative Commons Attribution International License (CC BY 4.0).

http://creativecommons.org/licenses/by/4.0/

cC) (7) Open Access

\begin{abstract}
Introduction: The diagnosis of pneumonia is usually made based on clinical manifestations and chest X-ray. The use of ultrasound in detecting pulmonary diseases in general, and especially consolidation syndrome has been demonstrated. The objective of this study was to determine the accuracy of thoracic ultrasound compared to chest X-ray in the diagnosis of infectious pneumonia in children. Methods: Children between 0 to 15 years were included in our study. The lung ultrasound results obtained were compared with those of the chest X-ray used as the reference. Our data were introduced into the EpiInfo 3.5.4 software and analyzed with the EpiInfo 3.5.4 and IBMSPSS Statistics version 20.0 softwares. Microsoft Office Excel 2016 was used to produce Charts. Continuous quantitative variables were presented. Cohen's Kappa concordance test was applied with confidence interval of 95\%. Results: 52 children were enrolled in the study. In imaging, the dominant sign was consolidation syndrome (75.0\%) of cases by chest radiography, and in $78.8 \%$ of cases by lung ultrasound $(\mathrm{p}<0.05)$. The chest radiograph was identified pleural effusion in 5 cases whereas lung ultrasound was identified in 6 cases. 3 patients with apparently normal chest X-rays had pulmonary ultrasounds suggestive of pneumonia. The degree of agreement between chest $\mathrm{X}$-ray and lung ultrasound in detecting lesions compatible with pneumonia
\end{abstract}


was satisfactory (kappa: 0.784) and excellent in the detection of pleural effusion (0.898). Conclusion: Our study demonstrated that lung echography is a non-ionizing and reliable tool in the diagnosis of childhood's pneumonia.

\section{Keywords}

Lung Ultrasound, Chest X-Ray, Pneumonia, Children, Yaoundé, Cameroon

\section{Introduction}

Pneumonia is a major cause of infant mortality worldwide. The diagnosis is usually based on the clinical presentations and findings on conventional chest $\mathrm{X}$-rays. The use of ultrasound scan to detect pulmonary diseases in general and particularly consolidation syndromes had been demonstrated. In Africa, few articles on its efficiency to detect pneumonia in children are published. The aim of this study was to determine the precision with which thoracic ultrasound scan can diagnose pneumonia in children relative to conventional chest X-rays.

\section{Materials and Method}

It was a cross-sectional, analytic study conducted in the Pediatric and Radiology Departments of the Yaoundé Gyneco-Obstetric and Pediatric Hospital (YGOPH), and the Mother-and-Child Centre (MCC) of the Chantal Biya's Foundation. The study was conducted for eight months, between the periods from October 2017 to May 2018.

Included in our study were children between 0 to 15 years old, who were either received at the out-patients consultations or admitted at the Pediatric departments of the YGOPH and the MCC of the Chantal Biya's Foundation with a clinical diagnosis of pneumonia that warrants radiologic confirmation, and for whom parents and guidance consented.

Due to the fact that we never had portable radiologic and ultrasound apparatuses to carrying bed-side scans, children with unstable vital signs were excluded from our study since they couldn't be displaced.

Children who were received at the out-patients or admitted with respiratory signs and symptoms caused by another lung disorders besides pneumonia were excluded from our study.

We study many variables: The socio-demographic variable (age and sex), the clinicals variables as respiratory sign, biologicals variables (hemogramm, C-reactive protein, hemocultur, pleural effusion), imaging variable as chest X-ray sign and ultrason finding.

Our procedure involved a consecutive non-exhaustive sampling based on the selection criteria during the period of data collection, and we used the central tendency comparism analysis. Our data were introduced into the EpiInfo 3.5.4 software and analyzed with the EpiInfo 3.5.4 and IBMSPSS Statistics version 20.0 
softwares. Microsoft Office Excel 2016 was used to produce Charts. Continuous quantitative variables were presented as means \pm standard deviations or medians (interquartile intervals), and categorical variables presented as portions (proportions in percentages). Cohen's Kappa concordance test was applied with confidence interval of $95 \%$.

\section{Results}

\subsection{Demographic, Clinical and Biologic Findings}

Our sample size consisted of 52 patients, with 34 males (65.4\%) and 18 females (34.6\%). The minimum age was 1 month and the maximum age was 23 month. The most represented age bracket was that between 2 to 24 months.

The prevalent clinical symptoms were fever 52 (100.0\%), cough 50 (96.2\%) and breathlessness $47(90.4 \%)$ with $55.8 \%$ having moderate respiratory distress. In $76.9 \%$ of cases, chest expansion was reduced, vocal transmission were increased in $55.8 \%$ of cases, dullness or subtle dullness in $92.3 \%$ of cases and breath sounds reduced in $82.7 \%$. The most perceived added breath sounds were fine crackles with a frequency of $75.0 \%$.

In terms of biochemistry, leukocytosis was the most frequent findings in full blood counts (94.2\%). Ninety-two point three percent (92.3\%) of cases presented with an elevated C-reactive protein levels, 9.6\% of children did blood culture and pleural fluid analysis test. Forty percent (40.0\%) of the blood culture test conducted were sterile. Le most isolated germ in both the blood culture and pleural fluid analysis was Mycobacterium Tuberculosis.

\subsection{Characteristic Findings on Imaging}

\subsubsection{Categories of Patients Based on Conventional X-Rays Findings}

According to the 52 patients who did the thoracic X-ray, 39 (75.0\%) of them presented the consolidation syndrome while, 11 (21.1\%) where normal. See Table 1 .

\subsubsection{Categories of Patients Based on Ultrasound Scan Findings} $41(78.8 \%)$ patients over 52 presented an alveolar syndrome at the chest ultrasonography. See Table 2.

Table 1. Categories of patients based on conventional X-rays findings.

\begin{tabular}{cc}
\hline Chest X-rays findings & Frequency and percentage (\%) \\
Normal & $11(21.1 \%)$ \\
Consolidation syndrome & $39(75.0 \%)$ \\
Interstitial syndrome & $8(15.4 \%)$ \\
Alveolo-interstitial syndrome & $8(15.4 \%)$ \\
Pleural syndrome & $5(9.6 \%)$ \\
Thickening of bronchial walls & $4(7.7 \%)$ \\
\hline
\end{tabular}


Table 2. Categories of patients based on ultrasound scan findings.

\begin{tabular}{cc}
\hline Ultrasound findings & Frequency and percentage (\%) \\
\hline Normal & $11(21.1 \%)$ \\
Alveolar syndrome & $41(78.8 \%)$ \\
Interstitial syndrome & $8(15.4 \%)$ \\
Alveolo-interstitial syndrome & $8(15.4 \%)$ \\
Pleural syndrome & $6(11.5 \%)$ \\
Pleural irregularity & $20(38.5 \%)$ \\
Average duration in minutes ( \pm standard deviation) & $8.5( \pm 2)$ \\
\hline
\end{tabular}

\subsection{Correlation between Conventional X-Rays and Ultrasound Scan}

\subsubsection{Correlation between the Two Modalities in Detecting Alveolar Syndrome}

Of the 52 children with suspected pneumonia at admission, 41 of them had findings that were comparable with those on lung ultrasonography. Standard chest radiographs confirmed pneumonia in 39 patients. There was a satisfactory degree of concordance between the standard X-rays and pulmonary ultrasound in detecting lesions comparable with pneumonia (Kappa: 0.784). See Table 3.

\subsubsection{Correlation between the Two Modalities in Detecting Pleural Effusion}

Six children presented with fluid pleural effusion at the ultrasound scan against 5 in chest X-rays, for which concordance is 0.898 between the two modalities (Table 4).

\section{Discussion}

\subsection{Limitations of our Study}

This study presents some shortcomings. Firstly, thoracic Computerized Tomography which is the gold standard imaging modality for diagnosing pneumonia was not used to compare findings on Chest X-rays and Ultrasound. This is because of the very high risk of radiation in CT scan with 300 to $400 \mathrm{x}$ that compared to conventional chest X-rays. Moreover, CT scan is expensive than X-rays [1].

Secondly, the same operator conducted the pulmonary ultrasound. Different findings could be gotten from a more or less experienced operator. Nonetheless, according to the "International Laison Committee for Pulmonary Ultrasonography", the pattern of experience increases relatively rapidly and simple with respect to other ultrasound modalities [2]. Shah et al. compared chest Ultrasound interpretations from inexperienced and experienced pediatricians and found out that there was improved performance linked to experience, however without demonstrating a statistically significant difference between the two categories of operators [3]. 
Table 3. Correlation between the two modalities in detecting alveolar syndrome.

\begin{tabular}{cccccccccc}
\hline \multicolumn{8}{c}{ Chest Ultrasonography } \\
\hline $\begin{array}{c}\text { Standard } \\
\text { Chest X-rays }\end{array}$ & Positive & Negative & Total & Sen (\%) & Sp (\%) & PPV (\%) & MPV (\%) & Kappa & P value \\
\hline Positive & 38 & 1 & 39 & 97.4 & 76.9 & 92.7 & 90.9 & 0.784 & 0.000 \\
Négative & 3 & 10 & 13 & & & & & & \\
Total & 41 & 11 & 52 & & & & & & \\
\hline
\end{tabular}

Table 4. Correlation between the two modalities in detecting pleural effusion.

\begin{tabular}{|c|c|c|c|c|c|c|c|c|c|c|}
\hline \multicolumn{11}{|c|}{ Chest Ultrasonography (Fluid pleural effusion) } \\
\hline $\begin{array}{c}\text { Standard chest } \\
\text { X-rays }\end{array}$ & PL & Yes & No & Total & $\mathrm{Se}(\%)$ & $\mathrm{Sp}(\%)$ & PPV (\%) & NPV (\%) & Kappa & $P$ value \\
\hline & Yes & 5 & 0 & 5 & 100.0 & 97.9 & 83.3 & 100.0 & 0.9 & 0.0 \\
\hline & No & 1 & 46 & 47 & & & & & & \\
\hline & Total & 6 & 46 & 52 & & & & & & \\
\hline
\end{tabular}

Thirdly, although the duration to perform the workups was reasonable enough ( $48 \mathrm{~h}$ ), it wasn't optimal. This could be justified by the fact that the same operator had to shuffle between the two sites of recruitment and performing the workups dependent on their availability.

Fourthly, ours sample size was small. This can be justified by the very limited time for our study. Boursiani et al. conducted a similar study with a sample size of 69 children over a period of 13 months [4]. Ianello et al. conducted a study with a sample size of 84 children over a period of 15 months [5].

\subsection{Demographic and Clinical Characteristics}

Our pool consisted of $65.4 \%$ boys. The average age was 14 months; the most represented age bracket was between 2 to 24 months giving $48.1 \%$ of patients. These results are similar to those reported by Omran et al. [6] who had $60.0 \%$ boys, the most represented age bracket was between 2 years. Tirdia et al. in their study had $65.5 \%$ boys and the average age children was 36 months [7]. The most present symptoms were fever (100.0\%), cough $(96.2 \%)$, and breathlessness (90.4\%). The most frequent signs were tachypnea (92.3\%), dullness $(92.3 \%)$ and reduced tactile fremitus $(80.2 \%)$. These results are similar to those reported by Yadav et al. who found $100.0 \%, 72.8 \%, 100.0 \%$ and $68.6 \%$ for fever, cough, breathlessness and tachypnea respectively [8].

\subsection{Biological Characteristics}

94.2\% of children had leukocytosis with $92.3 \%$ having a positive CRP test. These results were close to that obtained by Zayat et al. with $91.1 \%$ for positive CRP test [9]. $90.4 \%$ of patients never did blood culture tests. This can be explained by the high level of automedication, since doctors prescribed blood cultures only to 
patients with declining clinical conditions despite good drug compliance or, in cases of pleural effusion. Amongst the $9.6 \%$ of patients who did blood culture tests, $40.0 \%$ were sterile. So many factors could explain this relatively high level of sterile blood cultures especially the site of sample collection, and the conditions under sample collection. Given the financial challenges of parents, it is usually difficult to perform several blood cultures. In a study with 168 patients diagnosed of pneumonia, Wubbel et al. had all sterile blood cultures [10]. The most encountered germs in blood cultures and pleural effusion analysis was Mycobacterium Tuberculosis. These findings contradict the epidemiologic expectations whereby the primary causal germ of pneumonia in children is Streptococcus Pneumoniae. This can be explained by the fact that an empirical antibiotic therapy targeting common germs are usually commenced at the moment pneumonia is clinically suspected without blood culture test. Only in cases of treatment resistance to these antibiotic therapy that blood cultures and pleural fluid analysis is done if they were prescribed, reason for the low rate of these workups relative to the sample population. Given that Mycobacterium Tuberculosis is not sensitive to classical drugs, the tendency for these tests to be positive is then high.

\subsection{Conventional Chest X-Rays}

Amongst the 52 children in our study, consolidation syndrome was detected in 39 patients giving $75.0 \%$. These results are close to those reported by Caiulo et al. who had $79.4 \%$ of pneumonia detected by standard chest X-rays [11]. Tirdia et al. had $76.9 \%$ of alveolar syndrome detected by chest X-rays [7]. These findings were higher than that gotten by Boursiani et al. in their study where-in standard chest X-rays demonstrated a consolidation syndrome in 33.3\% of cases [4]. This difference can be due to the delay in consultations that is generally common in areas were the population is impoverished, added to the fact that there is a delay in appearance of radiographic signs with respect to the beginning of the diseases.

\subsection{Chest Ultrasonography}

In our study, thoracic ultrasound permitted us to detect consolidation syndrome in $78.8 \%$ of children with suspected pneumonia. The diagnostic performance of chest ultrasound in our study involved: Sensitivity (Se) of 97.4\%, Specificity (Sp) of $76.9 \%$, Positive Predictive Value (PPV) of 92.7\%, Negative Predictive Value (NPV) of $90.9 \%$. Similar findings had been reported by other authors. According to Yadav et al. chest ultrasound had a sensitivity of $98.0 \%$ and specificity of $64.7 \%$ for diagnosing acute community pneumonia in children [8]. The high sensitivity of chest ultrasound in detecting consolidation syndrome is of great value in clinical practice especially in the context of patient management with antibiotics, given that the syndrome is usually related to pneumonia caused by bacteria, while interstitial syndrome is linked to atypical bacteria or viruses.

On the contrary, we had a specificity of $76.9 \%$. Some studies reported similar 
findings, such as that of Ambroggio et al. [12] who had a specificity of $75.0 \%$ while comparing thoracic ultrasound with standard chest X-rays and CT scan in detecting consolidation syndrome. However, a good number of studies got higher specificities varying between 86.0 and 97.5\% [4] [7] [13] [14] [15] [16]. This could be due to the fact that the selection criteria, which involved entry diagnosis based on clinical signs wasn't specific, and the clinical evolution as well as exit diagnosis were never considered as judgment criteria together with the standard chest X-rays at the end of the study.

Thoracic ultrasound identified 6 cases of pleural effusion as against 5 for the standard chest X-rays, giving a sensitivity of $100.0 \%, 97.9 \%, 83.3 \%$, and $100.0 \%$. Hence, chest ultrasonography is an excellent tool to exclude fluid presence. These results were in line with those defined by the study from Yilmaz et al. in which conventional X-rays identified 4 cases of pleural effusion as against 5 in ultrasound [17]. While comparing thoracic ultrasound and standard X-rays to thoracic CT scan in detecting pleural effusion, sensitivity and specificity obtained were $80.0 \%$ and $78.0 \%$ respectively for ultrasound; $60.0 \%$ and $92.0 \%$ for $\mathrm{X}$-rays [12].

The average duration taken to realize a thoracic ultrasound was 8.5 minutes. This indicates that this modality is applicable in daily practice. Other studies demonstrated similar average test performance durations [11] [18] [19]. Shale et al. Reported an average performing duration of 8 minutes for inexperienced ultrasound operators ( $\leq 25$ ultrasound scans before the study) and 7 minutes for experienced operators ( $\geq 25$ ultrasound scans before the study).

\subsection{Diagnostic Correlation with Thoracic X-Rays and Ultrasound}

Chest ultrasound detected 3 cases of pneumonia non-visualized by the chest $\mathrm{X}$-rays. This may be due to the fact that standard chest does not detect small consolidations $(<1 \mathrm{~cm})$ as well as early consolidations. Among the positive $\mathrm{X}$-rays, a local lesion for pneumonia at the scapular area was not detected by the ultrasound scan. This could be explained by the difficulty of the chest echography to exploit the scapular region. The same challenge had been highlighted by other authors [20].

The concordance level between the two modalities was satisfactory (Kappa coefficient 0.784 ) for the diagnosis of pneumonia, and excellent (Kappa coefficient 0.898) for the detection of pleural effusion. In Urbankowsa's study [21], analysis of the concordance level demonstrated an excellent global concordance between thoracic ultrasound and X-rays in terms of pneumonia diagnosis (Cohen Kappa coefficient of 0.89 ). Such a good concordance indicates that in applying chest ultrasound, diagnosis will remain high while reducing children's exposure to ionizing radiations. In a case-control clinical trial conducted by Jones et al., in children with clinical suspicion of pneumonia, the use of chest ultrasound before the chest X-rays in a group of children reduced the use of chest $\mathrm{X}$-rays $38.8 \%$ relative to the control group. Amongst children who benefited 
from the chest ultrasound only, no missed pneumonia diagnosis nor undesirable findings was mentioned, indicating that it could be applicable to substitute chest $\mathrm{X}$-rays with chest ultrasonography while evaluating children with clinically suspected pneumonia [22].

\section{Conclusion}

At the end of our study, we could conclude that thoracic ultrasound is an applicable imaging modality in our context especially at the emergency services and health facilities without a radiographic apparatus. So chest ultrasound could be used as an alternative to chest X-rays and even used as a first choice test with aim to reduce child's exposure to ionizing radiations.

\section{Conflicts of Interest}

The authors declare no conflicts of interest regarding the publication of this paper.

\section{References}

[1] Linet, M.S., Kim, K.P. and Rajaraman, P. (2009) Children's Exposure to Diagnostic Medical Radiation and Cancer Risk: Epidemiologic and Dosimetric Considerations. Pediatric Radiology, 39, S4-S26. https://doi.org/10.1007/s00247-008-1026-3

[2] Volpicelli, G., Mussa, A., Garofalo, G., Cardinale, L., Casoli, G., Perotto, F., et al. (2006) Bedside Lung Ultrasound in the Assessment of Alveolar-Interstitial Syndrome. American Journal of Emergency Medicine, 24, 689-696. https://doi.org/10.1016/j.ajem.2006.02.013

[3] Shah, V., Tunik, M. and Tsung, J. (2013) Prospective Evaluation of Point-of-Care Ultrasonography for the Diagnosis of Pneumonia in Children and Young Adults. JAMA Pediatrics, 167, 119-125. https://doi.org/10.1001/2013.jamapediatrics.107

[4] Children, C., Boursiani, C., Tsolia, M. and Koumanidou, C. (2017) Lung Ultrasound as First-Line Examination for the Diagnosis of Community-Acquired Pneumonia in Children. Pediatric Emergency Care, 33, 62-66. https://doi.org/10.1097/PEC.0000000000000969

[5] Ianniello, S., Piccolo, C., Buquicchio, G., Trinci, M. and Miele, V. (2018) First-Line Diagnosis of Paediatric Pneumonia in Emergency: Lung Ultrasound (LUS) in Addition to Chest-X-Ray (CXR) and Its Role in Follow-Up. British Journal of Radiology, 89, Article ID: 20150998. https://doi.org/10.1259/bjr.20150998

[6] Johnson, J. and Kline, J. (2017) Intraobserver and Interobserver Agreement of the Interpretation of Pediatric Chest Radiographs. Emergency Radiology, 17, 285-290. https://doi.org/10.1007/s10140-009-0854-2

[7] Tirdia, P.R., Vajpayee, S., Singh, J. and Gupta, R.K. (2016) Accuracy of Lung Ultrasonography in Diagnosis of Community Acquired Pneumonia in Hospitalized Children as Compared to Chest X-Ray. International Journal of Contemporary Pediatrics, 3, 1026-1031. https://doi.org/10.18203/2349-3291.ijcp20162385

[8] Yadav, K., Awasthi, S. and Parihar, A. (2017) Lung Ultrasound Is Comparable with Chest Roentgenogram for Diagnosis of Community-Acquired Pneumonia in Hospitalised Children. Indian Journal of Pediatrics, 84, 499-504.

https://doi.org/10.1007/s12098-017-2333-1 
[9] Salah, R., Zayat, E., Bahbah, W., Abd, W. and Mousa, E. (2018) Lung Ultrasonography versus Chest X Ray for Diagnosing Pneumonia in Children with Fever and Respiratory Distress: A Prospective Blind Study. American Journal of Pediatrics, 4 , $15-20$.

[10] Wubbel, L., Muniz, L., Ahmed, A., Trujillo, M., Carubelli, C., McCoig, C., et al. (1999) Etiology and Treatment of Community-Acquired Pneumonia in Ambulatory Children. Pediatric Infectious Disease Journal, 18, 98-104. https://doi.org/10.1097/00006454-199902000-00004

[11] Caiulo, V., Gargani, L., Caiulo, S., Fisicaro, A., Moramarco, F., Latini, G., et al. (2013) Lung Ultrasound Characteristics of Community-Acquired Pneumonia in Hospitalized Children. Pediatric Pulmonology, 48, 280-287. https://doi.org/10.1002/ppul.22585

[12] Ambroggio, L., Sucharew, H., Rattan, M., O’Hara, S., Babcock, D., Clohessy, C., et al. (2016) Lung Ultrasonography: A Viable Alternative to Chest Radiography in Children with Suspected Pneumonia? Journal of Pediatrics, 176, 93-98.e7. https://doi.org/10.1016/j.jpeds.2016.05.033

[13] Yilmaz, H.L., Özkaya, A.K., SarıGökay, S., Tolu Kendir, Ö. and Şenol, H. (2017) Point-of-Care Lung Ultrasound in Children with Community Acquired Pneumonia. American Journal of Emergency Medicine, 35, 964-969. https://doi.org/10.1016/j.ajem.2017.01.065

[14] Pereda, M., Chavez, M., Hooper-Miele, C., Gilman, R., Steinhoff, M., Ellington, L., et al. (2015) Lung Ultrasound for the Diagnosis of Pneumonia in Children: A Meta-Analysis. Pediatrics, 135, 714-722. https://doi.org/10.1542/peds.2014-2833

[15] Ibrahim, M., Omran, A., Sayed, M., Bioumy, N. and Sharkawy, S. (2016) Diagnosis of Neonatal Transient Tachypnea and Its Differentiation from Other Causes of Neonatal Respiratory Distress Using Lung Ultrasound in Egyptian Neonates. American Journal of Perinatology, 33, A014. https://doi.org/10.1055/s-0036-1592385

[16] Principi, N., Esposito, A., Giannitto, C. and Esposito, S. (2017) Lung Ultrasonography to Diagnose Community-Acquired Pneumonia in Children. BMC, 17, 212. https://doi.org/10.1186/s12890-017-0561-9

[17] Cogliano, V., Baan, R., Straif, K. and Grosse, Y., Lauby-Secretan, B., El Ghissassi, F., et al. (2011) Preventable Exposures Associated with Human .Journal of the National Cancer Institute, 103, 1827-1839. https://doi.org/10.1093/jnci/djr483

[18] Shah, S.P., Epino, H., Bukhman, G., Umulisa, I., Dushimiyimana, J., Reichman, A., et al. (2009) Impact of the Introduction of Ultrasound Services in a Limited Resource Setting: Rural. BMC International Health and Human Rights, 9, 4. https://doi.org/10.1186/1472-698X-9-4

[19] Prithviraj, D., Reddy, B., Dodda, D. and Mazumder, M. (2017) Relevance of Lung Ultrasound in Diagnosis of Severe Neonatal Pneumonia. International Journal of Pediatrics, 4.

[20] Ianniello, S., Piccolo, C., Buquicchio, G., Trinci, M. and Miele, V. (2016) First-Line Diagnosis of Paediatric Pneumonia in Emergency: Lung Ultrasound (LUS) in Addition to Chest-X-Ray (CXR) and Its Role in Follow-Up. British Journal of Radiology, 89, 20150998. https://doi.org/10.1259/bjr.20150998

[21] Urbankowska, E., Krenke, K., Drobczyński, Ł., Korczyński, P., Urbankowski, T., Krawiec, M., et al. (2018) Lung Ultrasound in the Diagnosis and Monitoring of Community Acquired Pneumonia in Children. Respiratory Medicine, 109, 1207-1212. https://doi.org/10.1016/j.rmed.2015.06.011 
[22] Jones, B., Tay, E., Elikashvili, I., Sanders, J., Paul, A., Nelson, B., et al. (2016) Feasibility and Safety of Substituting Lung Ultrasonography for Chest Radiography When Diagnosing Pneumonia in Children: A Randomized Controlled Trial. Chest, 150, 131-138. https://doi.org/10.1016/j.chest.2016.02.643 